\title{
MINIMAL SURFACES AND MINIMIZERS OF THE GINZBURG-LANDAU ENERGY
}

\author{
O. SAVIN
}

\section{INTRODUCTION}

In this expository article we describe various properties in parallel for minimal surfaces and minimizers of the Ginzburg-Landau energy

$$
J(u)=\int \frac{1}{2}|\nabla u|^{2}+W(u) d x
$$

where $W$ is a double-well potential with minima at \pm 1 . Our goal is to present several results in both settings together with their common underlying ideas. Special interest is given to flatness theorems and their application to the regularity theory of minimal surfaces and 1D symmetry of global minimizers.

We start by briefly explaining the close relation between minimal surfaces and level sets of minimizers of $J$. Consider a minimizers $u$ of $J$ which is bounded by -1 and 1. The classical double-well potential $W$ to have in mind is $W(s)=\frac{1}{4}\left(1-s^{2}\right)^{2}$. In order for our ideas to become more transparent, later we will consider the discontinuous potential $W(s)=\chi_{(-1,1)}(s)$, where $\chi_{(-1,1)}$ represents the characteristic function of the interval $(-1,1)$.

The behavior of $u$ in large domains is given by the behavior of the rescaled functions $u_{\varepsilon}$ in $B_{1}$,

$$
u_{\varepsilon}(x):=u\left(\frac{x}{\varepsilon}\right)
$$

If $u$ minimizes $J$ in the ball $B_{\frac{1}{\varepsilon}}$ then $u_{\varepsilon}$ minimizes the rescaled energy $J_{\varepsilon}$ in $B_{1}$

$$
J_{\varepsilon}(v):=\int_{B_{1}} \frac{\varepsilon}{2}|\nabla v|^{2}+\frac{1}{\varepsilon} W(v) d x .
$$

We continue with a heuristic discussion about minimizers of $J_{\varepsilon}$. For a given function $v$, the main contribution in $J_{\varepsilon}(v)$ comes from the potential energy which is minimized when $v$ equals either 1 or -1 . Instant jumps from a region where $v=1$ to a region where $v=-1$ are not allowed since the kinetic energy $\int \frac{\varepsilon}{2}|\nabla v|^{2}$ would become infinite. However, it is useful to notice that if instead of the Dirichlet integral we would have the BV norm $\int \varepsilon|\nabla v|$ then such jumps are allowed and in this case the energy is minimized when the jumps occur along a minimal surface.

Clearly

$$
\int_{B_{1}} \frac{\varepsilon}{2}|\nabla v|^{2}+\frac{1}{\varepsilon} W(v) d x \geq \int_{B_{1}} \sqrt{2 W(v)}|\nabla v| d x
$$

and using the co-area formula

$$
|\nabla v| d x=d_{\mathcal{H}^{n-1}(\{v=s\})} d s
$$


the inequality becomes

$$
J_{\varepsilon}(v) \geq \int_{-1}^{1} \sqrt{2 W(s)} \mathcal{H}^{n-1}(\{v=s\}) d s .
$$

The energy $J_{\varepsilon}$ is then minimized by the function $v$ if every level set is a minimal surface and we have equality in the inequality above i.e $|\nabla v|=\frac{1}{\varepsilon} \sqrt{2 W(v)}$. This last equality gives

$$
v(x)=g_{0}\left(\frac{d_{\Gamma}(x)}{\varepsilon}\right),
$$

where $d_{\Gamma}(x)$ represents the sign distance to the 0 level set $\Gamma:=\{u=0\}$ and $g_{0}$ is the solution to the ODE

$$
g_{0}^{\prime}=\sqrt{2 W\left(g_{0}\right)}, \quad g_{0}(0)=0 .
$$

The function $g_{0}$ is in fact the unique minimizer of $J$ in 1D which is increasing.

In general the level sets of a function $v$ as above cannot be all minimal surfaces. However, if for example the 0 level set $\Gamma$ is minimal then the $s$-level sets are essentially minimal as long as $s$ is not too close to \pm 1 and $\varepsilon$ is small. On the other hand when $s$ is close to \pm 1 the weight $\sqrt{2 W(s)} d s$ becomes negligible. Thus such a function is "almost" a minimizer for $J_{\varepsilon}$. This suggests that the level sets of minimizers of $J_{\varepsilon}$ converge to a minimal surface as $\varepsilon \rightarrow 0$. The rigorous statement was proved by Modica in [12].

Theorem [Modica] There exists a subsequence $\varepsilon_{k} \rightarrow 0$ such that

$$
u_{\varepsilon_{k}} \rightarrow \chi_{E}-\chi_{\mathcal{C} E} \quad \text { in } L_{l o c}^{1}\left(B_{1}\right)
$$

and $E$ is a set with minimal perimeter in $B_{1}$ (i.e $\partial E$ is a minimal surface).

From the discussion above we see that in general the converse is also true i.e given a minimal surface $\partial E$ we can find a sequence of minimizers $u_{\varepsilon}$ converging to $\chi_{E}-\chi_{\mathcal{C} E}$. Therefore minimal surfaces and minimizers of $J_{\varepsilon}$ should have similar properties at least for small $\varepsilon$.

In Section 3 we will obtain as a consequence of the density estimates for minimizers that the convergence in the theorem above is in fact stronger than $L_{l o c}^{1}$ i.e. the level sets of $u_{\varepsilon_{k}}$ converge uniformly on compact sets to $\partial E$. Going back to a global minimizer $u$ of $J$, this implies that the blow-down sets $\varepsilon_{k}\{u=0\}$ converge uniformly to a minimal surface. It is a difficult problem to understand how well the original level set $\{u=0\}$ can be approximated by a minimal surface. For example in the case when $\partial E=\left\{x_{n}=0\right\}$ it is not clear wether or not $\{u=0\}$ stays at a bounded distance from $\partial E$. Also, given a nontrivial global minimal surface $\partial E$ it is not evident if there exists at least one minimizer for which $\{u=0\}$ stays at a bounded distance from $\partial E$. Such an example was given by Del Pino, Kowalczyk and Wei (see [6]) in dimension 9 which is the first dimension for which a nontrivial global minimal graph exists.

\section{Minimal Surfaces}

A very good reference for this section is the book of Giusti [11] where minimal surfaces are discussed in detail. Here we will introduce minimal surfaces and briefly discuss density estimates and monotonicity formula. Our goal is to give a different proof of the flatness theorem than the one of De Giorgi that appears in [11]. 
The approach of De Giorgi to studying minimal surfaces is to view them as boundaries of sets. Given a measurable set $E$ we define the perimeter of $E$ in an open set $\Omega \subset \mathbb{R}^{n}$ as the total variation of $\nabla \chi_{E}$ in $\Omega$, i.e.

$$
P_{\Omega}(E)=\int_{\Omega}\left|\nabla \chi_{E}\right|=\sup \left|\int_{E} \operatorname{div} g d x\right|
$$

where the supremum is taken over all vector fields $g \in C_{0}^{1}(\Omega)$ with $\|g\|_{L^{\infty}} \leq 1$.

We simply write $P(E)$ for the perimeter of $E$ in $\mathbb{R}^{n}$. Clearly, if $E$ is a set with $C^{1}$ boundary then $P(E)$ coincides with the classical notion of area of $\partial E$. A set $E$ which has locally finite perimeter is called a Caccioppoli set.

We say that a Caccioppoli set $E$ has minimal perimeter in $B_{1}$ if

$$
P_{B_{1}}(E) \leq P_{B_{1}}(F)
$$

for any set $F$ which coincides with $E$ outside $B_{1}$. The Plateau problem of finding a minimal surface in $B_{1}$ subject to boundary conditions can be thought in the following way:

Minimize $P(E)$ among all sets $E$ with $E \cap \mathcal{C} B_{1}=L \cap \mathcal{C} B_{1}$

where $L$ is a given Caccioppoli set. This set $L$ gives the boundary condition for the minimal surface and its shape outside a neighborhood of $\partial B_{1}$ clearly does not affect the minimizer $E$ in $B_{1}$. Existence of a minimizer follows easily from the compactness of $\mathrm{BV}$ functions in $L^{1}$. Below we give some important properties of such minimizers.

Compactness of minimizers. If $E_{n}$ is a sequence of minimal sets in $B_{1}$ then there exists a subsequence $E_{n_{k}}$ that converges to a minimal set $E$ i.e

$$
\chi_{E_{n_{k}}} \rightarrow \chi_{E} \quad \text { in } L_{l o c}^{1}\left(B_{1}\right) \text {. }
$$

Density estimates. Assume that $E$ has minimal perimeter in $B_{1}$ and $0 \in \partial E$. Since $E$ is merely a measurable set by $0 \in \partial E$ we understand that for any $\varepsilon>0$ we have $\left|B_{\varepsilon} \cap E\right|>0$ and $\left|B_{\varepsilon} \cap \mathcal{C} E\right|>0$. Then there exists a constant $c>0$ depending only on the dimension $n$ such that for all $r \in(0,1)$

$$
\frac{\left|E \cap B_{r}\right|}{\left|B_{r}\right|}>c, \quad \frac{\left|\mathcal{C} E \cap B_{r}\right|}{\left|B_{r}\right|}>c .
$$

We sketch the proof. Minimality implies

$$
P_{B_{1}}(E) \leq P_{B_{1}}\left(E \backslash B_{r}\right)
$$

or

$$
P_{B_{r}}(E) \leq \mathcal{H}^{n-1}\left(E \cap \partial B_{r}\right)
$$

hence

$$
P\left(E \cap B_{r}\right) \leq 2 \mathcal{H}^{n-1}\left(E \cap \partial B_{r}\right) .
$$

We denote $V(r)=\left|B_{r} \cap E\right|$ and we use the isoperimetric inequality

$$
c V(r)^{\frac{n-1}{n}} \leq P\left(E \cap B_{r}\right)
$$

to obtain

$$
c V(r)^{\frac{n-1}{n}} \leq V^{\prime}(r)
$$

or

$$
c \leq\left(V^{\frac{1}{n}}(r)\right)^{\prime}
$$

from which the first density estimate follows. 
Monotonicity formula. Assume $E$ is minimal and $0 \in \partial E$. Then

$$
\Phi_{E}(r)=\frac{P_{B_{r}}(E)}{r^{n-1}}
$$

is increasing in $r$. Moreover, $\Phi_{E}$ is constant if and only if $E$ is a cone, i.e $\lambda E=E$ for any $\lambda>0$. For simplicity we sketch the proof in the case when $\partial E$ is smooth.

Notice that $\Phi$ is invariant under dilations i.e

$$
\Phi_{\lambda E}(\lambda r)=\Phi_{E}(r)
$$

thus it suffices to show that $\Phi_{E}^{\prime}(1) \geq 0$ and that $E$ minimizes the perimeter in a set that contains $B_{1}$. Consider the set $F$ which coincides with $E$ outside $B_{1}$, contains a dilation of $E$ in $B_{\frac{1}{1+\varepsilon}}$ and is radial between $B_{1}$ and $B_{\frac{1}{1+\varepsilon}}$, i.e

$$
\chi_{F}(x)= \begin{cases}\chi_{E}(x) & 1<|x|, \\ \chi_{E}\left(\frac{x}{|x|}\right) & \frac{1}{1+\varepsilon} \leq|x| \leq 1, \\ \chi_{E}((1+\varepsilon) x) & |x|<\frac{1}{1+\varepsilon} .\end{cases}
$$

The inequality $P(E) \leq P(F)$ gives

$$
P_{B_{1}}(E) \leq(1+\varepsilon)^{-(n-1)} P_{B_{1}}(E)+P_{B_{1} \backslash B_{\frac{1}{1+\varepsilon}}}(F) .
$$

We let $\varepsilon \rightarrow 0$ and obtain

$$
(n-1) P_{B_{1}}(E) \leq \mathcal{H}^{n-2}\left(\partial E \cap \partial B_{1}\right) .
$$

On the other hand

$$
\Phi^{\prime}(1)=\int \frac{1}{\sqrt{1-(x \cdot \nu(x))^{2}}} d \mathcal{H}_{\partial E \cap \partial B_{1}}^{n-2}-(n-1) P_{B_{1}}(E),
$$

where $\nu(x)$ represents the normal to $E$ at $x$. Clearly we obtain $\Phi^{\prime}(1) \geq 0$ with equality if and only if $x \cdot \nu(x)=0$ for all $x \in \partial E \cap \partial B_{1}$.

Minimal cones. Assume $E$ is a minimal set and $0 \in \partial E$. Using compactness we see that there exists a sequence of blowup sets

$$
E_{k}:=\frac{1}{\varepsilon_{k}} E
$$

with $\varepsilon_{k} \rightarrow 0$ that converges in $L_{l o c}^{1}$ to a global minimal set $C$. From density estimates it follows that $0 \in \partial C$. Monotonicity formula implies that $\Psi_{C}$ is constant with value $\lim _{r \rightarrow 0} \Psi_{E}(r)$, hence $C$ is a minimal cone. We say that $C$ is a tangent cone to $E$ at 0 .

The minimal surfaces $\partial E_{k}$ converge in any compact set to $\partial C$ in the Hausdorff distance. Indeed, if $x_{0} \in \partial E_{k}$ is at distance $\delta$ from $\partial C$, then from the density estimates we find

$$
\int_{B_{\delta}\left(x_{0}\right)}\left|\chi_{E_{k}}-\chi_{C}\right| \geq c\left|B_{\delta}\right|
$$

Now the claim follows from the convergence of $E_{k}$ to $C$ in $L_{l o c}^{1}$.

Simons proved that up to dimension $n \leq 7$ the only global minimal cones $\partial C$ (and in fact the only global minimal surfaces) are the hyperplanes. Also it turns out that except on a small set of Hausdorff dimension $n-8$, the points of $\partial E$ have hyperplanes as tangent cones. 
Flatness theorems. In the remaining of this section we show how to "recover" smoothness of $\partial E$ near a point that has a half-space as a tangent cone at $0 \in \partial E$. At this point it is not clear wether different sequences of blow-ups of $E$ converge or not to the same limit. In view of the discussion above it suffices to prove the following flatness theorem.

Theorem [De Giorgi] Assume $E$ is minimal in $B_{1}, 0 \in \partial E$ and

$$
\partial E \cap B_{1} \subset\left\{\left|x_{n}\right| \leq \varepsilon_{0}\right\}
$$

with $\varepsilon_{0}(n)$ small depending only on $n$. Then $\partial E$ is an analytic surface in $B_{1 / 2}$.

The difficulty of the theorem lies in the fact that $\partial E$ cannot be written as a graph. De Giorgi's proof used monotonicity formula and approximation of $\partial E$ by harmonic functions. We will give a proof based on Harnack inequality and viscosity solutions methods. First we give a different version of the theorem above known as improvement of flatness.

Theorem [Improvement of flatness] Assume $E$ is minimal in $B_{1}, 0 \in \partial E$ and

$$
\partial E \cap B_{1} \subset\left\{\left|x_{n}\right| \leq \varepsilon\right\}
$$

with $\varepsilon \leq \epsilon_{0}(n)$. Then there exists a unit vector $\nu_{1}$ such that

$$
\partial E \cap B_{r_{0}} \subset\left\{\left|x \cdot \nu_{1}\right| \leq \frac{\varepsilon}{2} r_{0}\right\}
$$

where $r_{0}$ is a small universal constant.

This theorem implies that $\partial E$ is a $C^{1, \alpha}$ graph in $B_{3 / 4}$. To see this we apply the theorem inductively and we obtain unit vectors $\nu_{k}$ such that

$$
\partial E \cap B_{r_{0}^{k}} \subset\left\{\left|x \cdot \nu_{k}\right| \leq \frac{\varepsilon}{2^{k}} r_{0}^{k}\right\}
$$

This gives

$$
\left|\nu_{k+1}-\nu_{k}\right| \leq C\left(r_{0}\right) \frac{\varepsilon}{2^{k}}
$$

hence $\nu_{k} \rightarrow \nu(0)$ and moreover

$$
\left|\nu_{k}-\nu(0)\right| \leq C \frac{\varepsilon}{2^{k}}
$$

We obtain

$$
\partial E \cap B_{r_{0}^{k}} \subset\left\{x \cdot \nu(0) \leq C \frac{\varepsilon}{2^{k}} r_{0}^{k}=C \varepsilon r_{0}^{k(1+\alpha)}\right\},
$$

which implies that $\partial E$ is a differentiable surface at 0 with normal $\nu(0)$. Applying this argument at all points in $\partial E \cap B_{3 / 4}$ we see that $\partial E$ is in fact a $C^{1, \alpha}$ surface.

Once the surface is a $C^{1, \alpha}$ graph, then we can apply Schauder's estimates for the minimal surface equation satisfied by the graph and obtain that in $B_{1 / 2}, \partial E$ is smooth and in fact analytic.

The flat setting allows us to obtain the improvement of flatness theorem from the following weaker result.

Theorem [Harnack inequality] Assume $E$ is minimal in $B_{1}$ and

$$
\partial E \cap B_{1} \subset\left\{\left|x_{n}\right| \leq \varepsilon\right\}
$$


with $\varepsilon \leq \epsilon_{1}(n)$. Then $\partial E \cap B_{1 / 2}$ is either included in $\left\{x_{n} \leq \varepsilon(1-\eta)\right\}$ or in $\left\{x_{n} \geq-\varepsilon(1-\eta)\right\}$, where $\eta>0$ is a small universal constant.

Assume for the moment that Harnack inequality holds. We will prove the improvement of flatness theorem by compactness.

Assume by contradiction the statement is not true. Then we can find a sequence of minimal surfaces $\partial E_{k}$ which satisfy the hypothesis with $\varepsilon_{k} \rightarrow 0$ for which the conclusion does not hold. At each point $x_{0} \in \partial E_{k} \cap B_{1 / 2}$ we apply Harnack inequality and obtain that the oscillation of the set $\partial E_{k} \cap B_{1 / 2}\left(x_{0}\right)$ in the $x_{n}$ direction is less than $2 \varepsilon_{k}(1-\eta / 2)$. We apply Harnack inequality repeatedly as long as the hypothesis is satisfied. We obtain that for all $m$ such that

$$
\varepsilon_{k} 2^{m}(1-\eta)^{m-1}<\epsilon_{1}(n)
$$

the oscillation of the set

$$
\partial E_{k} \cap B_{2-m}\left(x_{0}\right)
$$

in the $x_{n}$ direction is less than $2 \varepsilon_{k}(1-\eta / 2)^{m}$. Clearly, $m \rightarrow \infty$ as $\varepsilon_{k} \rightarrow 0$.

We dilate this picture by a factor $\frac{1}{\varepsilon_{k}}$ in the $x_{n}$ direction. The sets

$$
A_{k}:=\left\{\left(x^{\prime}, \frac{x_{n}}{\varepsilon_{k}}\right) \mid\left(x^{\prime}, x_{n}\right) \in \partial E_{k} \cap B_{1}\right\}
$$

are included in $\left\{\left|x_{n}\right| \leq 1\right\}$. Moreover, for each $m$ as above the oscillation of $A_{k}$ in $\left|x^{\prime}-x_{0}^{\prime}\right| \leq 4^{-m}$ is less than $2(1-\eta / 2)^{m}$. By Arzela-Ascoli theorem we can assume, by passing if necessary to a subsequence, that $A_{k}$ converges in Hausdorff distance to the graph of a Hölder continuous function $\left(x^{\prime}, w\left(x^{\prime}\right)\right)$ in $\left\{\left|x^{\prime}\right| \leq 1 / 2\right\}$.

Next we show that $w$ is harmonic in the viscosity sense. Assume $P\left(x^{\prime}\right)$ is a quadratic polynomial whose graph touches by below the graph of $w$ at some point. Then $A_{k}$ touches a translation of $P$ which implies that $\partial E_{k}$ touches the graph of $\varepsilon_{k} P\left(x^{\prime}\right)+c$ at some interior point. Clearly $\partial E_{k}$ satisfies the minimal surface equation in the viscosity sense hence

$$
\varepsilon_{k} \triangle P+\varepsilon_{k}^{3}\left(|\nabla P|^{2} \Delta P-(\nabla P)^{T} D^{2} P \nabla P\right) \leq 0
$$

at the contact point. We let $\varepsilon_{k} \rightarrow 0$ and obtain $\triangle P \leq 0$.

Since $w$ is harmonic, $w(0)=0$ (since $\left.0 \in A_{k}\right)$ and $|w| \leq 1$, we find that

$$
\left|w\left(x^{\prime}\right)-x^{\prime} \cdot \nabla w(0)\right| \leq \frac{r_{0}}{4} \quad \text { if }\left|x^{\prime}\right| \leq 2 r_{0},
$$

provided that $r_{0}$ is chosen small, universal. This easily implies that $\partial E_{k}$ satisfies the conclusion of the Theorem for large $k$, contradiction.

Now it remains to prove Harnack inequality. This will follow easily from the next lemma. We denote points in $\mathbb{R}^{n}$ as $x=\left(x^{\prime}, x_{n}\right)$ with $x^{\prime} \in \mathbb{R}^{n-1}$. Also the $n-1$ dimensional ball of center $x_{0}^{\prime}$ and radius $r$ are denoted by $\mathcal{B}_{r}\left(x_{0}^{\prime}\right)$.

Lemma . Assume $\partial E \cap B_{1} \subset\left\{x_{n} \geq 0\right\},(0, \varepsilon) \in \partial E$ and fix $\delta>0$. There exist constants $C$ universal, and $C(\delta), \epsilon(\delta)$ depending on $\delta$ such that if $\varepsilon \leq \epsilon(\delta)$ then the set

$$
\partial E \cap\left\{x^{\prime} \in \mathcal{B}_{1 / 3},\left|x_{n}\right|<C(\delta) \varepsilon\right\}
$$

projects along $e_{n}$ into a set of $\mathcal{H}^{n-1}$ measure greater than $\mathcal{H}^{n-1}\left(\mathcal{B}_{1 / 3}\right)-C \delta$.

We sketch the proof of the lemma in two steps. 
Step 1: We show that in each cylinder $\left\{x^{\prime} \in \mathcal{B}_{\delta}\left(x_{0}^{\prime}\right)\right\}$, with $x_{0}^{\prime} \in \mathcal{B}_{1 / 3}$ there exists a point of $\partial E$ that stays at distance less than $C(\delta) \varepsilon$ from $\left\{x_{n}=0\right\}$.

It is easy to construct an explicit Lipschitz function $\varphi: \mathcal{B}_{2 / 3} \rightarrow \mathbb{R}$ such that $\varphi=0$ on $\partial \mathcal{B}_{2 / 3}, \varphi=C(\delta)$ in $\mathcal{B}_{\delta}, \varphi$ is strictly subharmonic in $\mathcal{B}_{2 / 3} \backslash \mathcal{B}_{\delta}$ and $\varphi>1$ in $\mathcal{B}_{1 / 3}$. Notice that if $\varepsilon<\epsilon(\delta)$ is small then $\varepsilon \varphi$ is a strict subsolution to the minimal surface equation in $\mathcal{B}_{2 / 3} \backslash \mathcal{B}_{\delta}$. Consider translations of the graph $\Phi_{\varepsilon}$ of $\varepsilon \varphi$ by vectors $y$ with $y^{\prime}=x_{0}^{\prime}$. If all points of $\partial E$ in the cylinder $\left\{x^{\prime} \in \mathcal{B}_{\delta}\left(x_{0}^{\prime}\right)\right\}$ were above $\left\{x_{n}=C(\delta) \varepsilon\right\}$ then, by sliding these graphs from below, we find that $\Phi_{\varepsilon}+x_{0}^{\prime}$ also lies below $\partial E$. This contradicts $(0, \varepsilon) \in \partial E$, and our claim is proved.

Step 2: We obtain the measure estimate by sliding balls $B_{r}(y)$ with $y^{\prime} \in \mathcal{B}_{\frac{1}{3}-4 \delta}$ from below in the vertical direction till they touch $\partial E$. By choosing the radius

$$
r:=\frac{2 \delta^{2}}{C(\delta) \varepsilon}
$$

it follows from Step 1 that all contact points occur in the set

$$
\left\{x^{\prime} \in \mathcal{B}_{1 / 3}, 0 \leq x_{n} \leq 2 C(\delta) \varepsilon\right\} .
$$

We study the map that associates to each contact point $x \in \partial E$ its corresponding center $y(x)$. Assume for simplicity that $\partial E$ is smooth in a neighborhood of such a contact point $x$. Since $y(x)=x-r \nu(x)$, where $\nu(x)$ denotes the normal to $\partial E$ at $x$ we see that the differential of this map equals

$$
D_{x} y(x)=I-r I I(x)
$$

where $I I(x)$ is the second fundamental form of $\partial E$ at $x$. On the other hand the mean curvature at $x$ is 0 , i.e $\operatorname{tr} I I(x)=0$ and moreover, since $\partial E$ has a tangent ball of radius $r$ from below at $x$ we have $I I(x) \geq-\frac{1}{r} I$. This gives

$$
\left|\operatorname{det} D_{x} y(x)\right| \leq 1 \text {. }
$$

This implies that infinitesimally the $\mathcal{H}^{n-1}$ measure of the contact points is less than the $\mathcal{H}^{n-1}$ measure of their corresponding centers. Finally we remark that the tangent plane to $\partial E$ at $x$ is parallel to the tangent plane to the surface of centers at $y(x)$. Therefore the statement above remains valid if we project the sets along the $e_{n}$ direction. In conclusion the $\mathcal{H}^{n-1}$ measure of the $e_{n}$-projection of the contact points is greater than $\mathcal{H}^{n-1}\left(\mathcal{B}_{\frac{1}{3}-4 \delta}\right)$ and the lemma is proved.

Now we can prove Harnack inequality. If we assume by contradiction that in $B_{1 / 2}, \partial E$ comes $\varepsilon \eta$ close from both constraint planes $\left\{x_{n}= \pm \varepsilon\right\}$ then we can apply the lemma above a number of times (with $\varepsilon \eta$ instead of $\varepsilon$ ) and conclude that both the $e_{n}$-projections of

$$
\partial E \cap\left\{x \in B_{1 / 2}, x_{n} \geq-\varepsilon(1-C(\delta) \eta)\right\}, \quad \partial E \cap\left\{x \in B_{1 / 2}, x_{n} \leq \varepsilon(1-C(\delta) \eta)\right\}
$$

have $\mathcal{H}^{n-1}$ measure greater than $\mathcal{H}^{n-1}\left(\mathcal{B}_{1 / 2}\right)-C \delta$. If $C(\delta) \eta<1$ then the sets are disjoint and this implies that

$$
P_{B_{1 / 2}}(E) \geq 2 \mathcal{H}^{n-1}\left(\mathcal{B}_{1 / 2}\right)-C \delta .
$$

On the other hand minimality of $E$ and $\partial E \cap B_{1} \subset\left\{\left|x_{n}\right| \leq \varepsilon\right\}$ clearly implies

$$
P_{B_{1 / 2}}(E) \leq \mathcal{H}^{n-1}\left(\mathcal{B}_{1 / 2}\right)+C \varepsilon
$$

We reach a contradiction by choosing $\delta$ small universal. 
Remarks: Our proof is based on the fact that $\partial E$ satisfies the mean curvature equation in the viscosity sense. The only place where we used minimality of $\partial E$ is in the last argument.

The same ideas can be applied to obtain estimates for flat solutions to more general elliptic equations or free boundary problems (see [14]). Roughly speaking, this method gives that flat solutions are as regular as the solutions of the linearized equation.

\section{Minimizers of $J$}

We consider minimizers of the energy functional

$$
J_{\Omega}(u)=\int_{\Omega} \frac{1}{2}|\nabla u|^{2}+\chi_{\{|u|<1\}} d x
$$

for functions $u$ that take values in the set $[-1,1]$. If $B_{r+2} \subset \Omega$ then we can compare $u$ with the compact perturbation

$$
w=\min \{u, h\} \quad \text { with } \quad h(x):=\min \left\{(|x|-r)^{+}-1,1\right\} .
$$

We easily obtain

$$
J_{B_{r}}(u) \leq C r^{n-1},
$$

which implies that the set $\{|u|<1\} \cap B_{r}$ has measure less than $\mathrm{Cr}^{n-1}$.

From the classical results of Alt and Caffarelli on the one-phase problem (see [3]) it follows that minimizers are uniformly Lipschitz in $B_{r}$,

$$
\triangle u=0 \text { in }\{|u|<1\},
$$

and $u$ satisfies the free boundary condition

$$
|\nabla u|=1 \quad \text { on } \quad \partial\{|u|<1\} .
$$

This last condition is understood in the viscosity sense, i.e. at any free boundary point $x_{0} \in \partial\{|u|<1\}$ that can be touched by a tangent ball included either in $\{|u|<1\}$ or in its complement, $u$ has a linear expansion of the form

$$
u(x)=\left(\left(x-x_{0}\right) \cdot \nu_{x_{0}}\right)^{+}+o\left(\left|x-x_{0}\right|\right), \quad \text { for some }\left|\nu_{x_{0}}\right|=1 .
$$

Density estimates. We present the density estimates obtained by Cordoba and Caffarelli [4]. Assume $u(0)=0$. There exists $c$ universal such that

$$
\frac{\left|\{u>0\} \cap B_{r}\right|}{\left|B_{r}\right|} \geq c,
$$

for any ball $B_{r} \subset \Omega$, with $r \geq C$ universal.

For each $r \geq 1$ denote by

$$
V(r):=\left|\{u>0\} \cap B_{r}\right|, \quad a(r):=\left|\{|u|<1\} \cap B_{r}\right| .
$$

We use the comparison function $w$ from the beginning of the section and denote by $K$ the closure of the open set $\{u>w\}$. Clearly

$$
J_{K}(u) \leq J_{K}(w),
$$

and, as in the introduction, the coarea formula gives

$$
\sqrt{2} \int_{-1}^{1} \mathcal{H}^{n-1}(\{v=s\} \cap K) \leq J_{K}(v),
$$


for any function $v$ with values in $[-1,1]$. For each $s \in(-1,1)$ we have

$$
\partial\{w<s<u\}=(\{u=s\} \cup\{w=s\}) \cap K
$$

and by the isoperimetric inequality

$$
|\{w<s<u\}|^{\frac{n-1}{n}} \leq \mathcal{H}^{n-1}(\{u=s\} \cap K)+\mathcal{H}^{n-1}(\{w=s\} \cap K) .
$$

Since for $s<0$,

$$
V(r)=\left|\{u>0\} \cap B_{r}\right| \leq|\{w<s<u\}|
$$

the inequalities above imply

$$
c V(r)^{\frac{n-1}{n}} \leq J_{K}(w)
$$

Also $J_{K}(u) \leq J_{K}(w)$ gives

On the other hand

$$
a(r) \leq J_{K}(w)
$$

$$
\begin{aligned}
\frac{1}{2} J_{K}(w) & \leq|\{w>-1\} \cap K| \leq\left|\{u>-1\} \cap\left(B_{r+2} \backslash B_{r}\right)\right| \\
& \leq V(r+2)-V(r)+a(r+2)-a(r) .
\end{aligned}
$$

In conclusion

$$
c\left(a(r)+V(r)^{\frac{n-1}{n}}\right) \leq V(r+2)+a(r+2)-(V(r)+a(r)) .
$$

From the universal Lipschitz estimate we have $a(r) \geq a(1) \geq c_{0}$ universal, hence the function

$$
f(r)=V(r)+a(r)
$$

satisfies

$$
c f(r)^{\frac{n-1}{n}} \leq f(r+2)-f(r), \quad f(1) \geq c_{0},
$$

which clearly implies

$$
f(r) \geq c r^{n} .
$$

Now the density estimate follows from the inequality $a(r) \leq \mathrm{Cr}^{n-1}$ deduced at the beginning of the section.

Asymptotic behavior. As a consequence of the density estimates we obtain that the level sets of $u$ are asymptotically flat at $\infty$ at least in low dimensions. If $u: \mathbb{R}^{n} \rightarrow[-1,1]$ is a minimizer then, by Modica's theorem, the rescalings $u_{\varepsilon_{k}}(x)=$ $u\left(x / \varepsilon_{k}\right)$ satisfy

$$
u_{\varepsilon_{k}} \rightarrow \chi_{E}-\chi_{\mathcal{C} E} \quad \text { in } L_{l o c}^{1}\left(\mathbb{R}^{n}\right) .
$$

Then the density estimates imply, as in the minimal surface case, that the level sets

$$
\left\{u_{\varepsilon_{k}}=0\right\}=\varepsilon_{k}\{u=0\}
$$

converge uniformly on compact sets to $\partial E$. Since $\partial E$ is a global minimal surface, then $\partial E$ is a hyperplane if $n \leq 7$, say $\partial E=\left\{x_{n}=0\right\}$. Then $\{u=0\}$ is asymptotically flat at $\infty$, i.e. there exist sequences $\theta_{k}, l_{k}$ with $l_{k} \rightarrow \infty, \theta_{k} / l_{k} \rightarrow 0$ such that

$$
\{u=0\} \cap B_{l_{k}} \subset\left\{\left|x_{n}\right| \leq \theta_{k}\right\} .
$$

We obtain the same conclusion for other $n$ under various extra assumptions on the 0 level set. For example, if $n=8$ it suffices to assume that $\{u=0\}$ is a graph over $\mathbb{R}^{n-1}$ in the $e_{n}$ direction. Moreover, if we assume that this graph grows at most linearly at $\infty$ then the conclusion holds in any dimension $n$. 
Flatness theorems. Next we present the flatness theorems for level sets of minimizers of $J$. The corresponding Harnack inequality is the following:

Theorem [Harnack inequality] Assume that $u$ is a minimizer of $J$ in the cylinder $\left\{\left|x^{\prime}\right|<l, \quad\left|x_{n}\right|<l\right\}$ and the 0 level set satisfies

$$
0 \in\{u=0\} \subset\left\{\left|x_{n}\right|<\theta\right\} .
$$

For any $\theta_{0}>0$ there exists $\epsilon\left(\theta_{0}\right)$ such that if

$$
\frac{\theta}{l}<\epsilon, \quad \theta \geq \theta_{0}
$$

then

$$
\{u=0\} \cap\left\{\left|x^{\prime}\right|<l / 2\right\} \subset\left\{\left|x_{n}\right|<\theta(1-\eta)\right\},
$$

where $\eta$ is a small constant depending only on $n$.

The difference with the minimal surface case is the appearance of new constants $\theta_{0}$ and $\epsilon\left(\theta_{0}\right)$. This is because in our setting the problem is no longer invariant under dilations. For example by taking $\epsilon<\theta_{0} / C$ we can always assume that $l>C$ and therefore the level sets of $u$ are long enough to capture the behavior of minimal surfaces.

The proof of Harnack inequality is similar to the one for minimal surfaces. We just point out the main ideas leaving out most of the details. The key step is to have a parallel version for the Lemma in Section 2.

We first introduce a family of sliding surfaces that replace the spheres $\partial B_{r}(y)$ of Step 2. Consider the parabola

$$
P_{y, a}:=\left\{x_{n+1}=\frac{1}{2 r}|x-y|^{2}+a\right\}
$$

and define its center

$$
Y=\left(y, y_{n+1}\right) \in \mathbb{R}^{n+1}, \quad y_{n+1}:=\frac{r}{2}+a .
$$

Whenever $y_{n+1} \in(-1,1)$ we define

$$
S(Y, r):=P_{y, a} \cap\left\{\left|x_{n+1}\right|<1\right\}
$$

the surface obtained by intersecting the parabola with the strip $\left|x_{n+1}\right| \leq 1$.

Notice that the plane $x_{n+1}=y_{n+1}$ intersects $S(Y, r)$ on the $n$-dimensional sphere $\partial B_{r}(y)$ and the slope of $S(Y, r)$ on this sphere equals 1 . Since the slope of $S(Y, r)$ on the -1 (1) level set is less (greater) than 1 it follows that whenever we slide these surfaces by below in the $e_{n}$ direction, the first contact point with the graph of $u$ cannot occur at an interior free boundary point.

Let's denote such a contact point by $X=(x, u(x))$ and by $Y(X)$ its corresponding center. Then

$$
Y(X)=X-r\left(\nabla u(x), \frac{|\nabla u(x)|^{2}-1}{2}\right) .
$$

The surface described by the centers $Y(X)$ is parallel to the surface of the $X$ 's, hence

$$
\left|\operatorname{det} D_{X} Y(X)\right|=\left|\operatorname{det} D_{x} y(x)\right|=\left|\operatorname{det}\left(I-r D^{2} u(x)\right)\right| \leq 1
$$


where for the last inequality we used

$$
\triangle u=0, \quad D^{2} u(x) \leq \frac{1}{r} I .
$$

This implies that infinitesimally the $\mathcal{H}^{n}$ measure of the contact points $X$ is greater than the $\mathcal{H}^{n}$ measure of the corresponding centers $Y(X)$. Since the two surfaces $X$ and $Y(X)$ are parallel, the same statement holds for their projections along the $e_{n}$ direction. We are in the same situation as in Step 2 of the Lemma in Section 2. Thus, if we start with a family $S(Y, r)$ with $\left(y^{\prime}, y_{n+1}\right) \in A \subset \mathbb{R}^{n}$ and slide them in the $e_{n}$ direction then the set of contact points projects along $e_{n}$ in a set of $\mathcal{H}^{n}$ measure greater than $\mathcal{H}^{n}(A)$.

In order to reproduce Step 1 of the Lemma we first need to obtain bounds for all level sets of $u$ from the location of the 0 level set. For example if $u<0$ below $\left\{x_{n}=0\right\}$ in the cylinder $\left\{\left|x^{\prime}\right|<l,\left|x_{n}\right|<l\right\}$ then the results of Caffarelli (see [3]) for the one-phase problem imply that the free boundary where $u=-1$ (and therefore any level sets) is above $\left\{x_{n}=-C\right\}$. In fact we can bound $u$ by a small translation of the $1 \mathrm{D}$ solution that has $x_{n}=0$ as 0 level set :

$$
\{u=s\} \text { is above }\left\{x_{n}=s-\frac{C}{l}\right\} \text { in the cylinder }\left|x^{\prime}\right|<l / 2 .
$$

To see this one needs to construct an explicit function $h$ which is radially symmetric and defined in an annulus around the sphere of radius $l / 4$ with the following properties:

a) $h$ increases in the radial direction from -1 to 1 and is continuous

b) $h$ is smooth except on the 0 level set where $\nabla h$ has a jump discontinuity

c) $\triangle h<0$ at all points where $h \neq 0$

d) $|\nabla h|<1$ when $h=-1$ and $|\nabla h|>1$ when $h=1$

e) $h=0$ is a sphere of radius $l / 4$ and $h=s$ is in a $C / l$ neighborhood of the sphere of radius $l / 4+s$.

Clearly, when we slide the graph of $h$ from below in the $e_{n}$ direction, the first contact point with the graph of $u$ occurs on the 0 level set. Since $\{u=0\}$ is above $\left\{x_{n}=0\right\}$ we obtain the desired bounds.

Now one can argue the same as in Section 2 provided that $l$ is large enough so that the error above $C / l \ll \theta_{0} \leq \theta$ and $\theta / l$ is small. We simply take as a sliding barrier the signed distance to a surface $\left\{x_{n}=\tilde{\varphi}\left(x^{\prime}\right)\right\}$ and then truncate it at the -1 and 1 level sets. Here $\tilde{\varphi}$ is a rescaling of the function $\varphi$ from Step 1 .

Next we want to obtain an improvement of flatness theorem for level sets of minimizers by using compactness and Harnack inequality.

If $\{u=0\}$ is trapped in a flat cylinder $\left\{\left|x^{\prime}\right|<l,\left|x_{n}\right|<\theta\right\}$, then we map it in the fixed cylinder $\left\{\left|y^{\prime}\right|<1,\left|y_{n}\right|<\theta\right\}$ by the linear map

$$
y^{\prime}=x^{\prime} / l, \quad y_{n}=x_{n} / \theta .
$$

Harnack inequality gives compactness of these rescalings for sequences with $\theta / l \rightarrow 0$ and $\theta \geq \theta_{0}$. As before, it suffices to show that any limiting set $\left(y^{\prime}, w\left(y^{\prime}\right)\right)$ is the graph of a harmonic function. Thus we need a "viscosity version" of the theorem of Modica: given $\delta$ and a quadratic polynomial $P$ with $\triangle P>\delta,\{u=0\}$ cannot be touched from below by $x_{n}=\theta P\left(x^{\prime} / l\right)$, say at the origin, in the cylinder $\left|x^{\prime}\right|<\delta l$. 
To see this let

$$
\tilde{P}:=P-\frac{\delta}{4 n}\left|y^{\prime}\right|^{2}
$$

and define the function $\psi$ to be the signed distance (positive above and negative below) to the graph

$$
\Gamma:=\left\{x_{n}=\theta \tilde{P}\left(x^{\prime} / l\right)\right\}
$$

truncated at the levels \pm 1 . Since

$$
\triangle \psi(x)=-\kappa(x)
$$

where $\kappa(x)$ is the mean curvature of the parallel surface to $\Gamma$ passing through $x$, and $\triangle \tilde{P}>\delta / 2$ we see that $\psi$ is superharmonic if $\theta / l$ is sufficiently small.

On the other hand, since $x_{n}=\theta P\left(x^{\prime} / l\right)$ is below $\{u=0\}$, one can obtain bounds for all the other level sets as in the proof of Harnack inequality and conclude that

$$
u<\psi \quad \text { on }\left|x^{\prime}\right|=\delta l
$$

provided that $C /(\delta l) \ll \delta \theta_{0}$. This implies that when we slide the graph of $\psi$ from below in the $e_{n}$ direction in the cylinder $\left|x^{\prime}\right| \leq \delta l$, the first contact point cannot occur neither on its boundary $\left|x^{\prime}\right|=\delta l$ nor in the interior since $\triangle \psi<0$ and moreover $|\nabla \psi|=1$ on the \pm 1 level sets.

In conclusion we obtain:

Theorem [Improvement of flatness] Let $u$ be a minimizer of $J$ in $\left\{\left|x^{\prime}\right|<l\right\} \times$ $\left\{\left|x_{n}\right|<l\right\}$, and assume that

$$
0 \in\{u=0\} \subset\left\{\left|x_{n}\right|<\theta\right\} .
$$

Then there exist small constants $0<\eta_{1}<\eta_{2}<1$ depending only on $n$ such that:

For $\theta_{0}>0$ there exists $\epsilon\left(\theta_{0}\right)>0$ depending on $n, \theta_{0}$ such that if

$$
\frac{\theta}{l} \leq \varepsilon, \quad \theta \geq \theta_{0}
$$

then

$$
\{u=0\} \cap\left\{\left|\pi_{\xi} x\right|<\eta_{2} l\right\} \subset\left\{|x \cdot \xi|<\eta_{1} \theta\right\}
$$

for some unit vector $\xi\left(\pi_{\xi}\right.$ denotes the projection along $\left.\xi\right)$.

As a consequence we obtain that global minimizers with asymptotically flat level sets at $\infty$ are one-dimensional i.e. their level sets are hyperplanes. Indeed, fix $\theta_{0}>0$ and choose $l, \theta$ large such that the hypotheses above are satisfied and

$$
\frac{\theta}{l}=\varepsilon<\epsilon\left(\theta_{0}\right) .
$$

We can apply the theorem repeatedly and stop when the height of the cylinder becomes less than $\theta_{0}$. Then the $\{u=0\}$ is trapped in a flat cylinder of height $\theta_{0}$ and radius $l_{0}$ with

$$
\frac{\theta_{0}}{l_{0}}<\frac{\varepsilon}{\eta_{1}}
$$

Since $\varepsilon$ can be taken arbitrary small we find that $\{u=0\}$ is trapped in an infinite strip of height $\theta_{0}$. We let $\theta_{0} \rightarrow 0$ and reach the desired result. Following the discussion on the asymptotic behavior of minimizers we obtain

Theorem. Minimizers $u: \mathbb{R}^{n} \rightarrow[-1,1]$ of $J$ are one-dimensional if $n \leq 7$. 
Theorem. Assume $\{u=0\}$ is a graph over $\mathbb{R}^{n-1}$ in the $e_{n}$ direction for a minimizer $u: \mathbb{R}^{n} \rightarrow[-1,1]$. Then

a) $u$ is one-dimensional if $n=8$.

b) $u$ is one-dimensional for any $n$ if the graph has at most linear growth at $\infty$.

In the last theorem it suffices to assume that $u$ is only a critical point for the energy $J$. This together with the graph assumption for $\{u=0\}$ imply that $u$ is monotone in the $e_{n}$ direction and $u$ is in fact a minimizer.

\section{Concluding REMARKS}

The problem of investigating one-dimensional symmetry for bounded solutions to the semilinear equation

$$
\triangle u=W^{\prime}(u)
$$

was proposed by De Giorgi in 1978. He conjectured that $e_{n}$-monotone solutions are one-dimensional at least in dimension $n \leq 8$ (with $W(s)=\left(1-s^{2}\right)^{2} / 4$ ). The conjecture was proved by Ghoussoub and Gui [10] for $n=2$ and Ambrosio and Cabre [1] for $n=3$.

Our approach gives one-dimensional symmetry of minimizers for $n \leq 7$. We also obtain one-dimensional symmetry for solutions of the semilinear equation above for $n \leq 8$ if we assume that $\{u=0\}$ is a graph over whole $\mathbb{R}^{n-1}$ instead of simply a graph as the $e_{n}$-monotonicity gives. However the proofs are more involved when the potential $W$ is different than $\chi_{(-1,1)}$ (see [13]). Then the surfaces $S(Y, r)$ are harder to construct and the measure estimate for $\left|\operatorname{det} D_{X} Y\right|$ holds with a constant smaller than 1 .

As we mentioned in the introduction Del Pino, Kowalczyk and Wei [6] provided a counterexample to De Giorgi's conjecture in dimensions $n \geq 9$.

There are further analogies between the two theories. Caffarelli and Cordoba proved in the context of minimizers the classical result of De Giorgi which states that Lipschitz minimal graphs are $C^{1, \alpha}$. As a consequence they obtain a result that was first proved by Barlow, Bass and Gui: De Giorgi's conjecture holds in any dimension if $\{u=0\}$ is a Lipschitz graph.

The proofs we presented use both variational techniques (density estimates, monotonicity formula, $\Gamma$-convergence) and non-variational techniques (sub and supersolutions, measure estimates). They are quite general and can be applied to more complicated settings such as the $p$-Laplace equation (see [16]), or for quasiminimizers (see [9]).

We also mention that one-dimensional symmetry was obtained in $2 D$ for monotone solutions of fully nonlinear equations (see [7], [8]). If we assume furthermore that one level set is Lipschitz then the result holds in any dimension (see [15]).

\section{REFERENCES}

[1] Ambrosio L., Cabre X., Entire solutions of semilinear elliptic equations in $\mathbb{R}^{3}$ and a conjecture of De Giorgi, J. American Math. Soc. 13, 2000, 725-739.

[2] Barlow M., Bass R., Gui C., The Liouville property and a conjecture of De Giorgi, Comm. Pure Appl. Math. 53 (2000), no. 8, 1007-1038.

[3] Caffarelli L., A Harnack inequality approach to the regularity of free boundaries. Part II: Flat free boundaries are Lipschitz, Comm. Pure Appl. Math. 42 (1989), no.1, 55-78. 
[4] Caffarelli L., Cordoba A., Uniform convergence of a singular perturbation problem. Comm. Pure Appl. Math. 48 (1995), no. 1, 1-12.

[5] Caffarelli L., Cordoba A., An elementary regularity theory of minimal surfaces, Differential Integral Equations, 6 (1993) no. 1-13.

[6] del Pino M., Kowalczyk, Wei J., On De Giorgi Conjecture in dimension $N \geq 9$. Preprint, arXiv:0806.3141

[7] De Silva D., Savin O., Symmetry of global solutions to a class of fully nonlinear elliptic equations in 2D, Indiana Univ. Math. Journal, Vol. 58, No.1 (2009) 301-315

[8] De Silva D., Valdinoci E., A fully nonlinear problem with free boundary in the plane, to appear in Annali Scuola Normale Superiore di Pisa.

[9] Farina A., Valdinoci E., Geometry of quasiminimal phase transitions, to appear in Calc. of Variations and PDEs.

[10] Ghoussoub N., Gui C., On a conjecture of De Giorgi and some related problems, Math. Ann. 311 (1998), 481-491.

[11] Giusti E., Minimal Surfaces and functions of bounded variation, Birkhauser Verlag, BaselBoston (1984).

[12] Modica L., $\Gamma$-convergence to minimal surfaces problem and global solutions of $\Delta u=2\left(u^{3}-u\right)$. Proceedings of the International Meeting on Recent Methods in Nonlinear Analysis (Rome, 1978), pp. 223-244, Pitagora, Bologna, 1979.

[13] Savin O., Regularity of flat level sets in phase transitions, Ann. of Math., 169 (2009) 41-78.

[14] Savin O., Small perturbation solutions for elliptic equations, Comm. Partial Differential Equations, 32, 557-578, 2007.

[15] Savin O., Entire solutions to a class of fully nonlinear elliptic equations ,Annali Scuola Normale Sup. Pisa, Cl. Sc. (5) Vol. II (2008), 369-405.

[16] Valdinoci E., Sciunzi B., Savin O., Flat level set regularity of $p$-Laplace phase transitions. Mem. Amer. Math. Soc. 182 (2006), no. 858.

Department of Mathematics, Columbia University, New York, NY 10027

E-mail address: savin@math.columbia.edu 